\title{
Combined effects of replacement of sucrose with D-tagatose and addition of different probiotic strains on quality characteristics of chocolate milk
}

\author{
M. Rouhi • R. Mohammadi • A. M. Mortazavian • \\ Z. Sarlak
}

Received: 18 May 2014 / Revised: 24 July 2014 / Accepted: 18 August 2014 /

Published online: 26 September 2014

(C) INRA and Springer-Verlag France 2014

\begin{abstract}
Nowadays, tendency to improve human nutrition and consume new healthful foods such as low-calorie and functional ones has been increased. In this study, effects of ratios of sucrose/D-tagatose (100:0, 0:100, or 50:50) as well as type of commercial probiotic strains (Lactobacillus acidophilus LAFTI L10, Lactobacillus casei LAFTI L26, Lactobacillus rhamnosus HN001, and Bifidobacterium animalis subsp. lactis LAFTI B94) on biochemical and microbiological characteristics, percent of residual sugar, color, and sensory attributes of synbiotic chocolate milk were investigated during 21 days of refrigerated storage $\left(5^{\circ} \mathrm{C}\right)$. The treatments inoculated with L. acidophilus, L. casei, L. rhamnosus, and B. lactis showed significantly higher biochemical and color changes compared to non-probiotic ones. The greatest viability at the end of storage was related to the treatment of D-tagatose with L. rhamnosus (T-R) as well as D-tagatose with L. casei (T-C). Although L. acidophilus, L. casei, and L. rhamnosus mostly tended to ferment D-tagatose, B. lactis did not substantially consume the mentioned sugar. In general, the treatments T-R, ST-R (sucrose and D-
\end{abstract}

\footnotetext{
M. Rouhi

Department of Food Science, Engineering and Technology, Faculty of Agricultural Engineering and Technology, College of Agriculture \& Natural Resources, University of Tehran, P.O. Box 4111, Karaj, Iran

R. Mohammadi $\cdot$ Z. Sarlak

Students' Research Committee, Department of Food Science and Technology, National Nutrition and Food Technology Research Institute, Faculty of Nutrition Sciences, Food Science and Technology, Shahid Beheshti University of Medical Sciences, P.O. Box 19395-4741, Tehran, Iran
}

\section{A. M. Mortazavian $(\bowtie)$}

Department of Food Science and Technology, National Nutrition and Food Technology Research Institute, Faculty of Nutrition Sciences, Food Science and Technology, Shahid Beheshti University of Medical Sciences, P.O. Box 19395-4741, Tehran, Iran e-mail: mortazvn@sbmu.ac.ir

A. M. Mortazavian e-mail: mortazvn@yahoo.com 
tagatose with L. rhamnosus), T-B (D-tagatose with B. lactis), and ST-B (sucrose and Dtagatose with $B$. lactis) were realized as the best ones in terms of probiotic viability, functional property of D-tagatose, and sensory attributes. In conclusion, D-tagatose could be successfully used as a natural sugar substitute with functional properties for probiotic chocolate milks enhancing their health benefits, but the proper selection of ratio of sucrose/D-tagatose and type of probiotic strain is recommended.

Keywords Chocolate milk $\cdot$ D-tagatose $\cdot$ Low-calorie $\cdot$ Probiotic $\cdot$ Synbiotic

\section{Introduction}

Foods that are not intended only to satisfy hunger and provide humans with necessary nutrients but also to provide medicinal characteristics (prevent nutrition-related diseases and increase physical and mental well-being of consumers) are called "functional foods" (Menrad 2003). One of the most promising ways for development of functional foods is using probiotics, prebiotics, and synbiotics.

Probiotics are special types of live healthful microorganisms which possess favorable impacts on animal and human host mainly via maintaining and/or improving microbial balance between harmful and beneficial microbiota, especially in the intestine (Korbekandi et al. 2011). They have an established role in reducing human illnesses, particularly gastrointestinal infections caused by deficient or compromised gut microbiota. Other therapeutic functions are attributed to probiotics such as anti-cholesterol activity, alleviation of lactose intolerance symptoms, promotion of beneficial immune responses, antimicrobial impact, anti-high blood pressure effect, and anti-carcinogenic and anti-mutagenic activities (Shah 2007). Recently, there have been reports on potential benefits of probiotics for human skin (Krutmann 2009) and against colds and flu (Leyer et al. 2009).

Probiotics should be alive to an adequate number in order to exert their positive effects on health of the host. This attribute is known as "viability," namely adequate number of live probiotic cells in a food product at time of consumption (Mortazavian et al. 2010). No general agreement has been established on the recommended and suggested levels ranging from $10^{6}$ to over $10^{7}$ cfu.mL ${ }^{-1}$ (Ferdousi et al. 2013; Korbekandi et al. 2011; Mohammadi et al. 2011a; Mortazavian et al. 2010, 2011). However, it is generally recommended that probiotic culture must be present in the product at minimum numbers of $10^{7} \mathrm{cfu}_{\mathrm{mL}}^{-1}$ (IDF 1992). With respect to dairy products, a therapeutic minimum of $10^{9}$ cfu.day $^{-1}$ and consumption of $100 \mathrm{~g}$ or ml should translate to a food containing at least $10^{7}$ cells per gram or milliliter (Reid 2001), which is in agreement with current Japanese recommendations (Ishibashi and Shimamura 1993). These suggestions have been made to compensate possible decline in concentration of probiotic organisms during processing and storage of a probiotic product as well as passing through upper and lower parts of the gastrointestinal tract.

The most typical food matrices (vehicles) for probiotic bacteria are different kinds of fermented milk products, especially yogurt (Gueimonde et al. 2004). During the past few years, diversity of probiotic foods has increased on the market. Nowadays, probiotics can be found in non-fermented dairy products such as milk (Shin et al. 2000), ice cream (Mohammadi et al. 2011a) and dairy dessert (Mortazavian et al. 2011), 
and non-dairy products such as fruit juices, cereal-based foods (Helland et al. 2004), and meat-based products (Rouhi et al. 2013).

In non-fermented liquid flavored milks, chocolate flavor is among the most popular ones (Yanes et al. 2002). Also, chocolate milk is an excellent sports recovery beverage and has a great amount of carbohydrates more than that of plain milk, along with protein, electrolytes, and other key nutrients, in quantities that commercial carbohydrate replacement drinks cannot match (Bayoumi et al. 2011).

D-tagatose, an epimer of D-fructose, has attracted a great deal of attention in recent years (used in nonchronic drugs, tooth paste, mouth wash, dietary supplements, and a wide variety of foods such as beverages, healthy foods, cereals, health bars, chocolate, candy, chewing gum, yogurt, soft drinks, and milk-based drink along with bakery and confectioneries) due to its health benefits and similar properties to sucrose (appropriate sweetness - $92 \%$ of sucrose in $10 \%$ solutions - with a sucrose-like taste and no cooling effect or aftertaste). It has been also known as a flavor enhancer and a low caloric value sugar with tooth-friendly property (because of not being fermented by oral microflora) (FDA 2003; Kim 2004; Levin 2002). In the USA, tagatose is qualified as generally recognized as safe (GRAS) for use in foods under FDA-regulated program (Rulis 2001). Joint FAO/WHO Expert Committee on Food Additives (JECFA) stated that there is no need to limit the allowed daily intake (ADI) of tagatose (JECFA 2004). D-tagatose naturally occurs in Sterculia setigera gum and is found in small quantities in various foods such as sterilized and powdered cow milk, hot cocoa, and a variety of cheese, yogurt, and other dairy products. It is a highly soluble sugar and stable at $\mathrm{pH} 2-7$ (Levin 2002).

One of the numerous health benefits attributed to D-tagatose is prebiotic effect (Bertelsen et al. 1999). While D-tagatose is not fermented by bacteria of the dental plaque, not even after repeated exposure, certain members of the more varied microbiota of the intestinal tract are able to utilize D-tagatose as a substrate. D-tagatose fermentation appears to be particularly widespread among lactobacilli, enterococci, and other lactic acid bacteria (Bertelsen et al. 2001). Studies on the prebiotic activity of D-tagatose confirmed that D-tagatose promotes the growth of lactobacilli and leads to an increased production of butyrate which is considered to have a beneficial trophic effect on the colonic mucosa (Jensen BB, Buemann B 1998; Venema et al. 2005).

No study was found on investigating survival of probiotic strains added to chocolate milk, especially prebiotic chocolate milk, during storage and its impact on attributes of the product. The hypothesis was that formulation of probiotic chocolate milk containing D-tagatose on the one hand would lead to a highly functional dairy product (synbiotic food with proper impact on probiotics viability); on the other hand, it could provide an acceptable vehicle for probiotic bacteria (with acceptable sensory properties).

\section{Materials and methods}

\subsection{Starter culture}

The DVS pouches of commercially lyophilized single probiotic cultures including Lactobacillus acidophilus LAFTI L10 (DSM, Australia), Lactobacillus casei LAFTI 
L26 (DSM, Australia), Lactobacillus rhamnosus HN001 (Danisco, Denmark), and Bifidobacterium animalis ssp. lactis (B. lactis) LAFTI B94 (Danisco, Denmark) were used. These starter cultures are widely used in the dairy industry. The cultures were maintained according to the manufacturer's instructions until used.

\subsection{Sample preparation}

Milk with $15.0 \%$ dry matter was formulated using reconstituted skim milk powder (Anchor, New Zealand) and sterilized distilled water. Other ingredients including 1.0\% alkalized cocoa powder with fat content of 10-12\% (Schokinag, Germany) and $0.03 \%$ K-carrageenan (Sigma, USA) were added. Then, three treatments with different ratios of sucrose/D-tagatose (100:0, 50:50, and 0:100) were produced. In 100:0 treatments, 6.5\% of sucrose (Chem-Lab, Belgium) was added to chocolate milk. Considering relative sweetness of D-tagatose (0.92), 7.06\% of D-tagatose (Damhert, Belgium) was added to 0:100 treatments and $3.25 \%$ of sucrose and $3.53 \%$ of D-tagatose were added to $50: 50$ treatments. After heat treatment $\left(90{ }^{\circ} \mathrm{C}, 15 \mathrm{~min}\right)$, homogenization (2 stages, 200 and 50 bars at $90{ }^{\circ} \mathrm{C}$ ), and cooling to $15^{\circ} \mathrm{C}$, the treatments were inoculated in a hygienic condition with one of the probiotic single strain cultures (L. acidophilus L10, L. casei L26, L. rhamnosus HN001, or B. lactis B94) at initial concentration of $10^{8} \mathrm{cfu}_{\mathrm{mL}}^{-1}$. Also, the treatments without inoculated probiotic were produced as control. Chocolate milk was packed in a hygienic condition and stored for 21 days at refrigerated temperature $\left(5^{\circ} \mathrm{C}\right)$. The treatments with 100:0, 0:100, and 50:50 ratios of sucrose/Dtagatose were nominated as S, T, and ST, respectively. Those inoculated with L. acidophilus L10, L. casei L26, L. rhamnosus HN001, and B. lactis B94 were identified as A, C, R, or B, respectively. Therefore, 15 treatments were produced (S, T, and ST as controls, S-A, T-A, ST-A, S-C, T-C, ST-C, S-R, T-R, ST-R, S-B, T-B, and $\mathrm{ST}-\mathrm{B}) . \mathrm{pH}$, titrable acidity, redox potential, and viability of probiotics were monitored at day 0 as well as per 7-day intervals during 21 days of the refrigerated storage. Percent of the residual sugars, color, and sensory properties was studied only at days 0 and 21 . The residual sugars and biochemical changes were indicators for monitoring metabolic activity of probiotics in chocolate milk.

\subsection{Microbiological analysis}

Serial decimal dilutions were prepared with Ringer's solution (Ringer's tablets from Merck, Germany). MRS agar medium (Merck, Germany) was used for enumeration of probiotics. The plates were incubated aerobically (for lactobacilli) and anaerobically (for bifidobacteria) at $37^{\circ} \mathrm{C}$ for at least $72 \mathrm{~h}$ (Mortazavian et al. 2010). Anaerobic conditions were produced using the GasPac system (Merck, Germany) in anaerobic jars.

\subsection{Biochemical analysis}

$\mathrm{pH}$ values and redox potential of the samples were measured at room temperature using a $\mathrm{pH}$ meter (MA235, Mettler, Toledo, Switzerland). Titrable acidity was determined according to the modified method of Mortazavian et al. (2010) after mixing $10 \mathrm{~mL}$ of the sample with $40 \mathrm{~mL}$ of distilled water and titrating with $0.1 \mathrm{~N} \mathrm{NaOH}$ using $0.5 \%$ phenolphthalein. 
Parameters of mean $\mathrm{pH}$ drop rate, mean acidity increase rate, and mean redox potential increase rate during storage time were calculated as follows (Mohammadi et al. 2011b):

$$
\left.\mathrm{pH} \text { drop rate }=(\text { final } \mathrm{pH} \text { value }- \text { initial } \mathrm{pH} \text { value }) /{\text { storage time }\left[\mathrm{pH}_{\text {value }}\right. \text { day }}^{-1}\right]
$$

Acidity increase rate

$$
=(\text { final acidity value }- \text { initial acidity value }) / \text { storage time }\left[\text { Dornic degree.day }{ }^{-1}\right]
$$

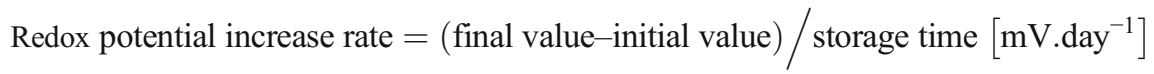

Time range of maximum $\mathrm{pH}$ drop $\left(t_{\max -\mathrm{pH}-\mathrm{D}}\right):$ 7-day time interval during refrigerated storage, in which the greatest $\mathrm{pH}$ decline is observed [day-day]

\subsection{Chemical analysis}

Lactose, sucrose, and D-tagatose were determined according to the method of Bogdanov and Baumann (1988). After filtering the solution, sugar content was determined by HPLC with a column of $4.6 \mathrm{~mm}$ internal diameter and $250 \mathrm{~mm}$ length, containing amine-modified silica gel with 5-7 $\mu \mathrm{m}$ particle size, Develosil (Nomura Chemical Co., Japan). Column and detector temperature were $30{ }^{\circ} \mathrm{C}$, mobile phase was degassed acetonitrile:water $(80: 20, v / v)$, and flow rate and sample volume were $1.3 \mathrm{~mL} \cdot \mathrm{min}^{-1}$ and $10 \mu \mathrm{L}$, respectively. Peaks were identified on the basis of their retention times. Standards of lactose, sucrose, and D-tagatose were supplied from Sigma (USA). Quantification was performed according to the external standard method on peak areas or peak heights. Determinations were performed on a Waters HPLC (USA) using Waters Refractive Index detector (USA). Percent recoveries were regarded in calculating percentage of sugars. Percent decrease in each type of sugar $(X)$ during the storage was calculated as follows:

$$
\text { Percent decrease of } X=\% X_{\text {day } 0}-\% X_{\text {day } 21}
$$

\subsection{Color analysis}

Color was measured using a Hunter Lab colorimeter (ColorFlex, Model no. 45/0, Hunter Lab Reston, Virginia, USA). 'L'=lightness is expressed as dark to light color. Green to red is expressed by ' $a$ ' value, and blue to yellow is expressed by ' $b$ ' value. Three readings of each parameter were taken for each sample. $C$ values, which were calculated using $C=\left(a^{2}+b^{2}\right)^{1 / 2}$ denote cumulative measurement of chromaticity or saturation, as determined by $a$ and $b$ values. $\Delta E$ (total difference) values were

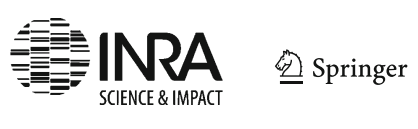


computed using $\Delta E=\left(\Delta L^{2}+\Delta a^{2}+\Delta b^{2}\right)^{1 / 2}$ where $\Delta L=L_{\text {day } 21}-L_{\text {day } 0}$ and the same was for $\Delta a$ and $\Delta b$. $\Delta E$ values provide cumulative measurement of total color changes as determined by changes in $L, a$, and $b$ values during 21 days of storage.

\subsection{Sensory analysis}

Sensory testing was conducted in compliance with "Committee of Morality in Research" in "National Nutrition and Food Technology Research Institute= NNFTRI" (Iran) and the panelists were requested to complete the human subjects' consent form. Twelve panelists were selected based on interest, time availability, and knowledge of dairy associated with sensory parameters. The panelists were selected from among faculty members, staff, and students of the NNFTRI. The panel was composed of eight male and four female individuals with mean age of 27 years old. The samples $(100 \mathrm{~mL})$ were served at $5 \pm 2{ }^{\circ} \mathrm{C}$ in $150 \mathrm{~mL}$ crystalline plastic cups fitted with white plastic lids and labeled with 3 -digit codes to remove bias. The packages were shaken uniformly before the analysis in order to re-suspend any settled cocoa particles in the bottom. The panelists individually evaluated five chocolate milks per session individually in an odor-free room under white fluorescent lights ( $70 \mathrm{ft} . \mathrm{c}^{-1}$ intensity) on a white Lab bench dedicated to sensory analysis.

Sensory evaluation was carried out at ambient temperature about $2 \mathrm{~h}$ before or after meals. Potable water was available for palate cleansing. The panelists received a tray containing five samples, a glass of water, an evaluation form, and a pencil. They were allowed to rest between trays to prevent fatigue. Chocolate milk formulations were compared using "scoring methodology" (Drake 2009). Sensory attributes included flavor (taste and odor), mouthfeel, appearance (color, visible purity, presence of foreign matter, spots of mold, and phase separation), and total score. The panelists scored the samples using a five-point hedonic scale, where $0=$ unconsumable, $1=$ unacceptable, $2=$ acceptable, $3=$ satisfactory, and $4=$ excellent. To calculate total score with weighted average, the given numbers for each sensory parameter were multiplied by the relevant coefficients, namely 6 for flavor, 3.5 for mouthfeel, and 2 for appearance.

\subsection{Statistical analysis}

The experiments were performed in triplicate and interactive effect of the variables and the ranked orders of means were determined using two-way "Analysis of Variance (ANOVA test)" at significance level of " $P<0.05$ " from Minitab software. The design of study was "completely randomized design (Full Factorial)."

\section{Results and discussion}

\subsection{Biochemical characteristics during refrigerated storage}

According to Fig. 1a-c, redox potential was very low and negative at the beginning of storage, which could be attributed to discharging much oxygen content from chocolate milk due to severe heat treatment as well as dissolving sugars. 
Table 1 shows mean $\mathrm{pH}$ drop rate, mean acidity increase rate, mean redox potential increase rate, time range of maximum $\mathrm{pH}$ drop, and initial and final titrable acidity in different treatments during the refrigerated storage. As shown in Fig. 1a-c and Table 1, non-probiotic treatments had no significant biochemical changes during the refrigerated storage $(P>0.05)$. In contrast, addition of probiotics made significant changes in the mentioned parameters. The amount of changes depended on strain of inoculated probiotic. The chocolate milk inoculated with $L$. acidophilus showed the greatest biochemical changes (mean $\mathrm{pH}$ drop rate, mean titrable acidity increase rate, and mean redox potential increase rate) during the storage $(P<0.05)$. Afterward, L. casei, L. rhamnosus, and B. lactis were placed, respectively. L. acidophilus and $L$. casei have high proteolytic activities (Sasaki et al. 1995) that improve their acidification rate. Proteolytic activities of probiotics are directly proportional to their acidification rate (Azcarate-Peril et al. 2005). Bifidobacteria are generally very poor fermentative probiotics, particularly at refrigerated temperatures (Lankaputhra et al. 1996) and cause slow pH decrease.

\section{a}
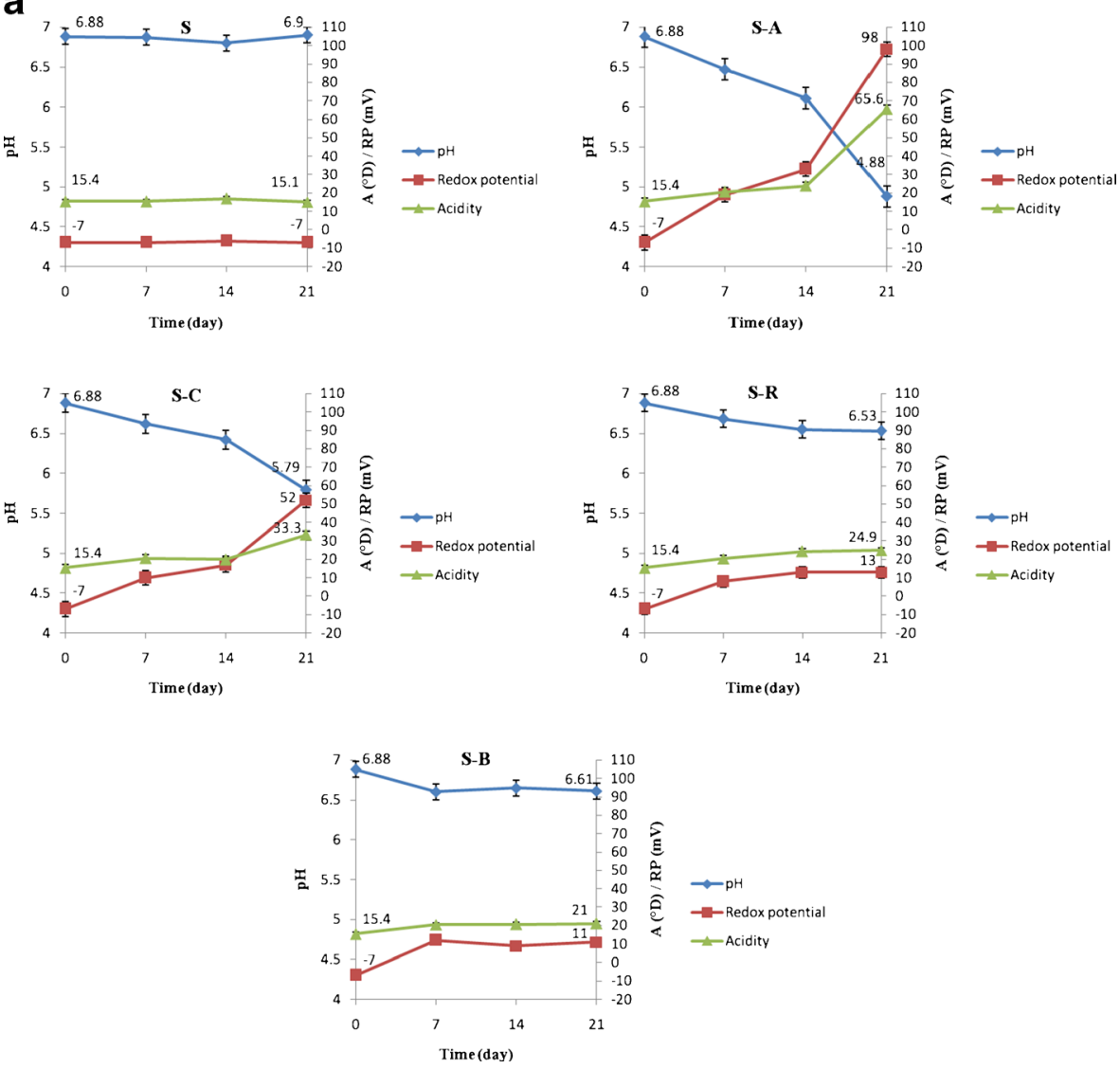

Fig. 1 a Changes in biochemical characteristics of treatments with no substitution of sucrose during 21 days of refrigerated storage. b Changes in biochemical characteristics of treatments with full substitution of sucrose with D-tagatose during 21 days of refrigerated storage. $\mathbf{c}$ Changes in biochemical characteristics of treatments with partial substitution of sucrose with D-tagatose (50:50) during 21 days of refrigerated storage 
Compared with sucrose, D-tagatose caused greater biochemical changes in all probiotic-containing treatments (lactobacilli), except bifidobacteria. The highest biochemical changes as well as highest final titrable acidity were related to T-A and the lowest ones were attributed to T-B. Therefore, D-tagatose stimulated growth and/or activity of lactobacilli probiotics more than sucrose. L. acidophilus was the most active probiotic in chocolate milk during the storage time. Time range of maximum $\mathrm{pH}$ drop $\left(t_{\text {max-pH-D }}\right)$ was different among probiotic chocolate milk during the storage. This parameter was day 14-21 of storage in treatments inoculated with L. acidophilus and L. casei, whereas day 0-7 in those with L. rhamnosus and B. lactis, meaning that L. rhamnosus and B. lactis showed peak of activity during 7 days of storage, followed by lack of activity until the end of this period. The aforementioned observations are illustrated in Fig. 1a-c.

3.2 Viability of probiotic bacteria during the refrigerated storage

Table 2 shows viability of probiotic microorganisms in different treatments during 21 days of the refrigerated storage. As represented by this table, viability of all probiotic

b
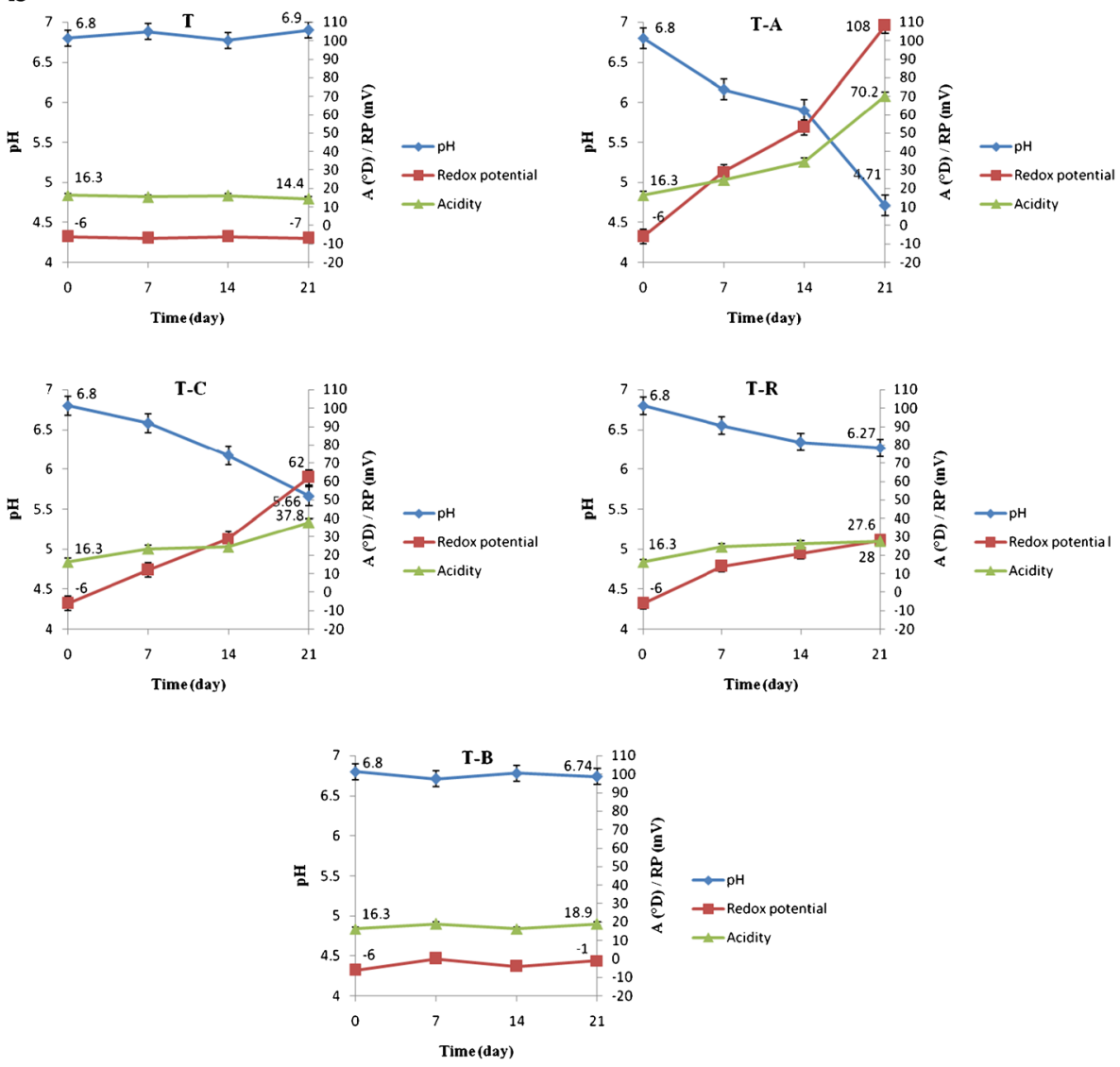

Fig. 1 continued. 
lactobacilli was significantly greater in tagatose-containing treatments, followed by mixture of sugars, which was in line with data of Table 1, in which D-tagatose caused greater $\mathrm{pH}$ drop rate and acidity increase rate in lactobacilli-containing treatments compared to sucrose (Section 3.1). For bifidobacteria, the highest and lowest viability was related to sucrose-containing and tagatose-containing treatments, respectively, which revealed that bifidobacteria was not active in the presence of D-tagatose compared to sucrose (Section 3.3). Therefore, the greatest viability throughout the storage time was related to D-tagatose with L. acidophilus (T-A), D-tagatose with L. casei (T-C), D-tagatose with L. rhamnosus (T-R), and sucrose with bifidobacteria (S-B).

From day 14 onwards, probably due to increase in titrable acidity and reduction in $\mathrm{pH}$ and sensitivity of probiotics to acidic media (Ventura et al. 2011), viability of lactobacilli in the presence of D-tagatose as well as bifidobacteria in the presence of sucrose considerably decreased. According to Table 1, this might be because time range of the maximum $\mathrm{pH}$ drop for L. acidophilus was day 14-21. Within this time interval, L. acidophilus showed the greatest biochemical changes during storage that led to harsher conditions and the highest decrease in viability after day 14. The present results were in agreement with those by Nighswonger et al. (1996) who reported that

\section{C}
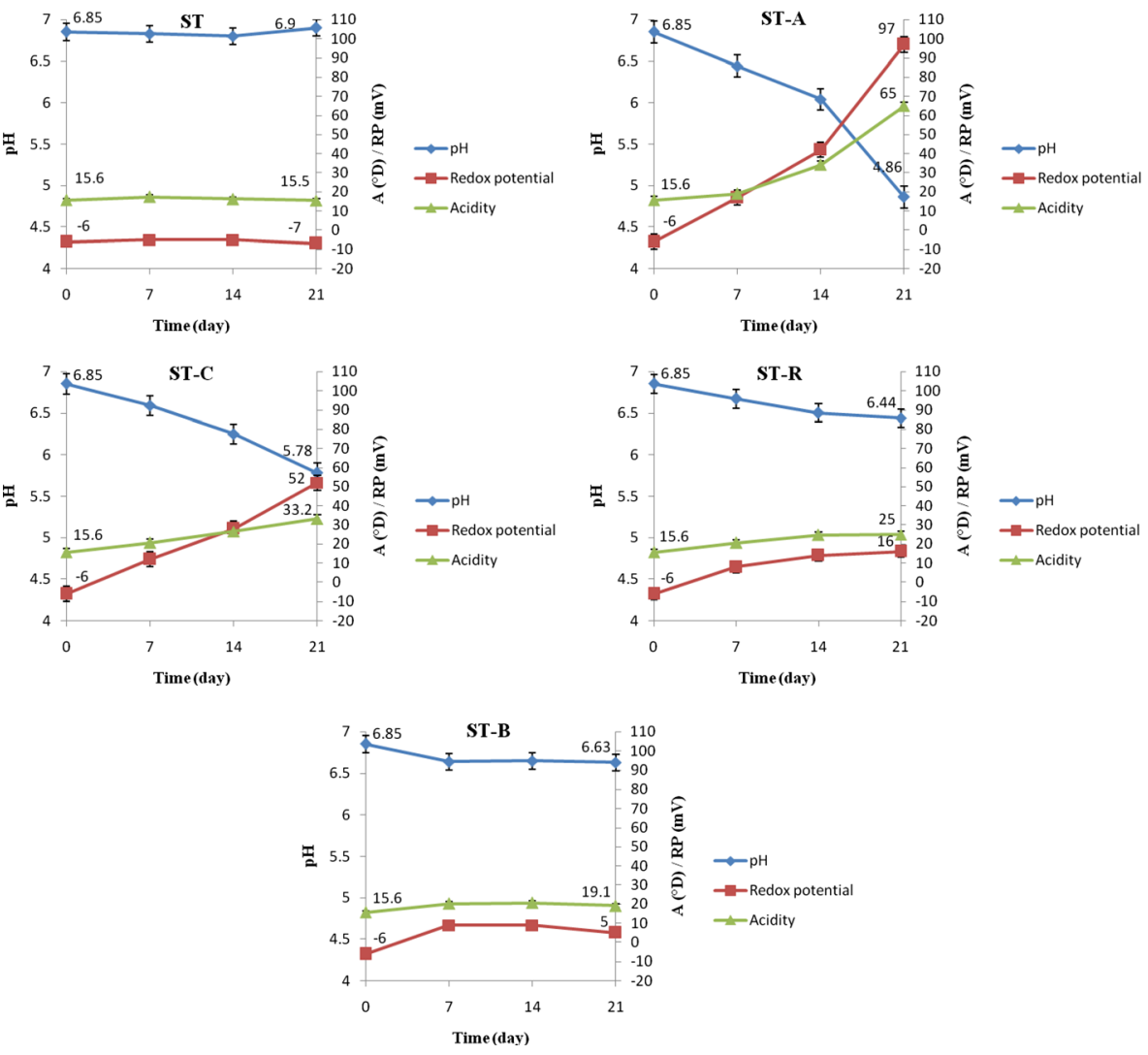

Fig. 1 continued. 
Table 1 Biochemical parameters of different treatments during 21 days of refrigerated storage

\begin{tabular}{|c|c|c|c|c|c|c|}
\hline Treatments $^{\mathrm{a}}$ & $\begin{array}{l}\text { M-pH-DR } \\
\left(1 . \text { day }^{-1}\right)\end{array}$ & $\begin{array}{l}\text { M-A-IR } \\
\left({ }^{\circ} \text { D.day }{ }^{-1}\right)\end{array}$ & $\begin{array}{l}\text { M-RP-IR } \\
\left(\mathrm{mV}^{\left.- \text {day }^{-1}\right)}\right.\end{array}$ & $\begin{array}{l}t_{\max -\mathrm{pH}-\mathrm{D}} \\
\text { (day-day) }\end{array}$ & $\begin{array}{l}\text { Initial titrable } \\
\text { acidity }\left({ }^{\circ} \mathrm{D}\right)\end{array}$ & $\begin{array}{l}\text { Final titrable } \\
\text { acidity }\left({ }^{\circ} \mathrm{D}\right)\end{array}$ \\
\hline S & $0.000 \mathrm{k}$ & $0.00 \mathrm{k}$ & 0.001 & - & $15.4 \mathrm{ab}$ & $15.1 \mathrm{i}$ \\
\hline $\mathrm{T}$ & $0.000 \mathrm{k}$ & $0.00 \mathrm{k}$ & 0.001 & - & $16.3 \mathrm{a}$ & $14.4 \mathrm{i}$ \\
\hline ST & $0.000 \mathrm{k}$ & $0.00 \mathrm{k}$ & 0.001 & - & $15.6 a b$ & $15.5 \mathrm{hi}$ \\
\hline S-A & $0.095 \mathrm{~b}$ & $2.39 \mathrm{~b}$ & $5.00 \mathrm{~b}$ & $14-21$ & $15.4 \mathrm{ab}$ & $65.6 \mathrm{~b}$ \\
\hline T-A & $0.099 \mathrm{a}$ & $2.56 \mathrm{a}$ & $5.42 \mathrm{a}$ & $14-21$ & $16.3 \mathrm{a}$ & $70.2 \mathrm{a}$ \\
\hline ST-A & $0.094 \mathrm{~b}$ & $2.35 \mathrm{c}$ & $4.90 \mathrm{c}$ & $14-21$ & $15.6 \mathrm{ab}$ & $65.0 \mathrm{~b}$ \\
\hline S-C & $0.051 \mathrm{~d}$ & $0.85 \mathrm{e}$ & $2.80 \mathrm{e}$ & $14-21$ & $15.4 \mathrm{ab}$ & $33.3 \mathrm{~d}$ \\
\hline $\mathrm{T}-\mathrm{C}$ & $0.054 \mathrm{c}$ & $1.02 \mathrm{~d}$ & $3.23 \mathrm{~d}$ & $14-21$ & $16.3 \mathrm{a}$ & $37.8 \mathrm{c}$ \\
\hline ST-C & $0.050 \mathrm{~d}$ & $0.83 \mathrm{e}$ & $2.76 \mathrm{e}$ & $14-21$ & $15.6 \mathrm{ab}$ & $33.2 \mathrm{~d}$ \\
\hline S-R & $0.016 \mathrm{~g}$ & $0.45 \mathrm{~g}$ & $0.95 \mathrm{~h}$ & $0-7$ & $15.4 \mathrm{ab}$ & $24.9 \mathrm{ef}$ \\
\hline T-R & $0.025 \mathrm{e}$ & $0.53 \mathrm{f}$ & $1.61 \mathrm{f}$ & $0-7$ & $16.3 \mathrm{a}$ & $27.6 \mathrm{e}$ \\
\hline ST-R & $0.019 f$ & $0.44 \mathrm{~g}$ & $1.04 \mathrm{~g}$ & $0-7$ & $15.6 \mathrm{ab}$ & $25.0 \mathrm{ef}$ \\
\hline S-B & $0.012 \mathrm{~h}$ & $0.26 \mathrm{~h}$ & $0.85 \mathrm{i}$ & $0-7$ & $15.4 \mathrm{ab}$ & $21.0 \mathrm{~g}$ \\
\hline T-B & $0.002 \mathrm{j}$ & $0.12 \mathrm{j}$ & $0.23 \mathrm{k}$ & $0-7$ & $16.3 \mathrm{a}$ & $18.9 \mathrm{gh}$ \\
\hline ST-B & $0.010 \mathrm{hi}$ & $0.16 \mathrm{i}$ & $0.52 \mathrm{j}$ & $0-7$ & $15.6 a b$ & $19.1 \mathrm{~g}$ \\
\hline
\end{tabular}

Means in the same column shown with different letters are significantly different $(P<0.05)$

${ }^{a} S$ treatments with ratio of the sucrose/D-tagatose: 100:0, $T$ those with ratio of the sucrose/D-tagatose: 0:100, $S T$ those with ratio of the sucrose/D-tagatose: 50:50, A L. acidophilus L10, C L. casei L26, $R$ L. rhamnosus HN001, B B. lactis B94, $M-p H-D R$ mean $\mathrm{pH}$ drop rate, $M-A-I R$ mean acidity increase rate, $M-R P-I R$ mean redox potential increase rate, $t_{\max -\mathrm{pH}-\mathrm{D}}$ time range of maximum $\mathrm{pH}$ drop

refrigerated storage stability of $L$. casei as an adjunct in fermented products was equal to or greater than that of strains of L. acidophilus tested on day 21 or 28 of refrigerated storage at 5 to $7^{\circ} \mathrm{C}$. Gilliland and Lara (1988) reported that some cells of L. acidophilus which failed to form colonies on the enumeration medium still possessed $\beta$ galactosidase activity. The lactobacilli contain either $\beta$-galactosidase and/or $\beta$ phosphogalactosidase as the enzyme responsible for enabling the organism to utilize lactose (Premi et al. 1972). Therefore, metabolic enzymes yielded from dead probiotics might increase fermentation ability of residual adapted and viable cells resulting in higher biochemical changes, which could cause more continuous declines in probiotics viability.

According to Fig. 1a-c and Table 1, time range of the maximum $\mathrm{pH}$ drop for biochemical changes in the treatments containing L. rhamnosus or B. lactis was on days 0-7 of storage. Antimicrobial effects of the organic acids produced during initial week of storage could be responsible for the afterward decline in viability of the mentioned probiotics. As can be observed in Table 2, the most decrease in viability of L. rhamnosus and B. lactis on days 14-21 of storage was related to D-tagatose- and sucrose-containing treatments, respectively. Another probable reason for considerable decrease of bifidobacteria viability could be increase in oxygen level of the product via permeation through the package during storage. If a minimum of $10^{7} \mathrm{cfu} \cdot \mathrm{mL}^{-1}$ viable counts of probiotics were considered standard level, all the treatments would have viability of higher than this level through 21 days of refrigerated storage. 
Table 2 Viable counts ( $\log$ cfu. $\mathrm{mL}^{-1}$ ) of probiotic microorganisms in different treatments during 21 days of refrigerated storage

\begin{tabular}{|c|c|c|c|c|}
\hline \multirow[t]{2}{*}{ Treatments $^{\mathrm{a}}$} & \multicolumn{4}{|c|}{ Storage time (day) } \\
\hline & 0 & 7 & 14 & 21 \\
\hline S-A & $8.02 \mathrm{aD}$ & $9.49 \mathrm{bB}$ & $9.97 \mathrm{dA}$ & $8.92 \mathrm{dC}$ \\
\hline T-A & $8.02 \mathrm{aD}$ & $9.89 \mathrm{aB}$ & $10.93 \mathrm{aA}$ & $9.04 \mathrm{bC}$ \\
\hline ST-A & $8.00 \mathrm{aD}$ & $9.51 \mathrm{bB}$ & $10.66 \mathrm{cA}$ & $8.94 \mathrm{cdC}$ \\
\hline $\mathrm{S}-\mathrm{C}$ & $8.01 \mathrm{aD}$ & $8.88 \mathrm{deC}$ & $9.54 \mathrm{eA}$ & $8.99 b c B$ \\
\hline $\mathrm{T}-\mathrm{C}$ & 7.98aD & $9.85 \mathrm{aB}$ & $10.83 \mathrm{bA}$ & $9.23 \mathrm{aC}$ \\
\hline ST-C & $8.02 \mathrm{aD}$ & $9.36 \mathrm{cB}$ & $9.99 \mathrm{dA}$ & 8.86deC \\
\hline S-R & 7.99aC & $8.49 \mathrm{fgB}$ & 8.75hiA & $8.79 \mathrm{eA}$ \\
\hline T-R & $8.02 \mathrm{aD}$ & $8.85 \mathrm{eC}$ & $9.32 \mathrm{fA}$ & $9.27 \mathrm{aAB}$ \\
\hline ST-R & $8.01 \mathrm{aC}$ & $8.55 \mathrm{fB}$ & $8.81 \mathrm{hA}$ & $8.82 \mathrm{eA}$ \\
\hline S-B & 7.99aB & $8.97 \mathrm{dA}$ & $8.95 \mathrm{gA}$ & $7.47 \mathrm{fC}$ \\
\hline T-B & $7.98 \mathrm{aB}$ & $8.44 \mathrm{ghA}$ & $7.78 \mathrm{kC}$ & $6.89 \mathrm{hD}$ \\
\hline ST-B & $8.00 \mathrm{aC}$ & $8.95 \mathrm{dA}$ & $8.51 \mathrm{jB}$ & $7.36 \mathrm{gD}$ \\
\hline
\end{tabular}

Means shown with different small and capital letters represent significant differences $(P<0.05)$ in the same columns (among the treatments) and rows (between the days of storage), respectively

${ }^{a} S$ treatments with ratio of the sucrose/D-tagatose 100:0, T those with 0:100, ST those with 50:50, AL. acidophilus L10, C L. casei L26, R L. rhamnosus HN001, B B. lactis B94

\subsection{Residual sugars in different treatments during 21 days of refrigerated storage}

Percentage of lactose, sucrose, and D-tagatose in different chocolate milk on day 0 and day 21 of storage as well as their decrease percent is shown in Table 3. Non-probiotic treatments in contrast to probiotic ones had no significant change in sugar amounts during the refrigerated storage. Therefore, addition of different probiotic microorganisms to chocolate milk led to significant decrease in the amount of all sugars. It should be noted that consumption pattern was different between the strains. Garro et al. (1999) studied the refrigerated shelf life of soymilk fermented with single cultures of Lactobacillus fermentum, L. casei, Streptococcus salivarius ssp. thermophilus, and Bifidobacterium longum. They reported that $L$. casei showed a high rate of sucrose utilization during the refrigerated storage at $4{ }^{\circ} \mathrm{C}$ for 28 days. According to results of the present study, both L. acidophilus and L. casei mostly tended to ferment D-tagatose, lactose, and sucrose, respectively. In the research by Garro et al. (1999), lactose and Dtagatose were not present in the environment. Also, L. casei CRL 207, different strain from the present study, was used. Moreover, soymilk matrix was very dissimilar to that of chocolate milk.

L. rhamnosus mostly fermented D-tagatose, sucrose, and lactose, respectively, and this order for B. lactis was sucrose, lactose, and D-tagatose for B. lactis. B. lactis did not significantly consume D-tagatose $(P>0.05)$. These results have been confirmed by several studies. Roy and Ward (1990) determined fermentation patterns of 20 Bifidobacterium strains by two methods: a carbohydrate utilization test based on acid production from a given range of sugars in the modified MRS broth (micromethod) and 


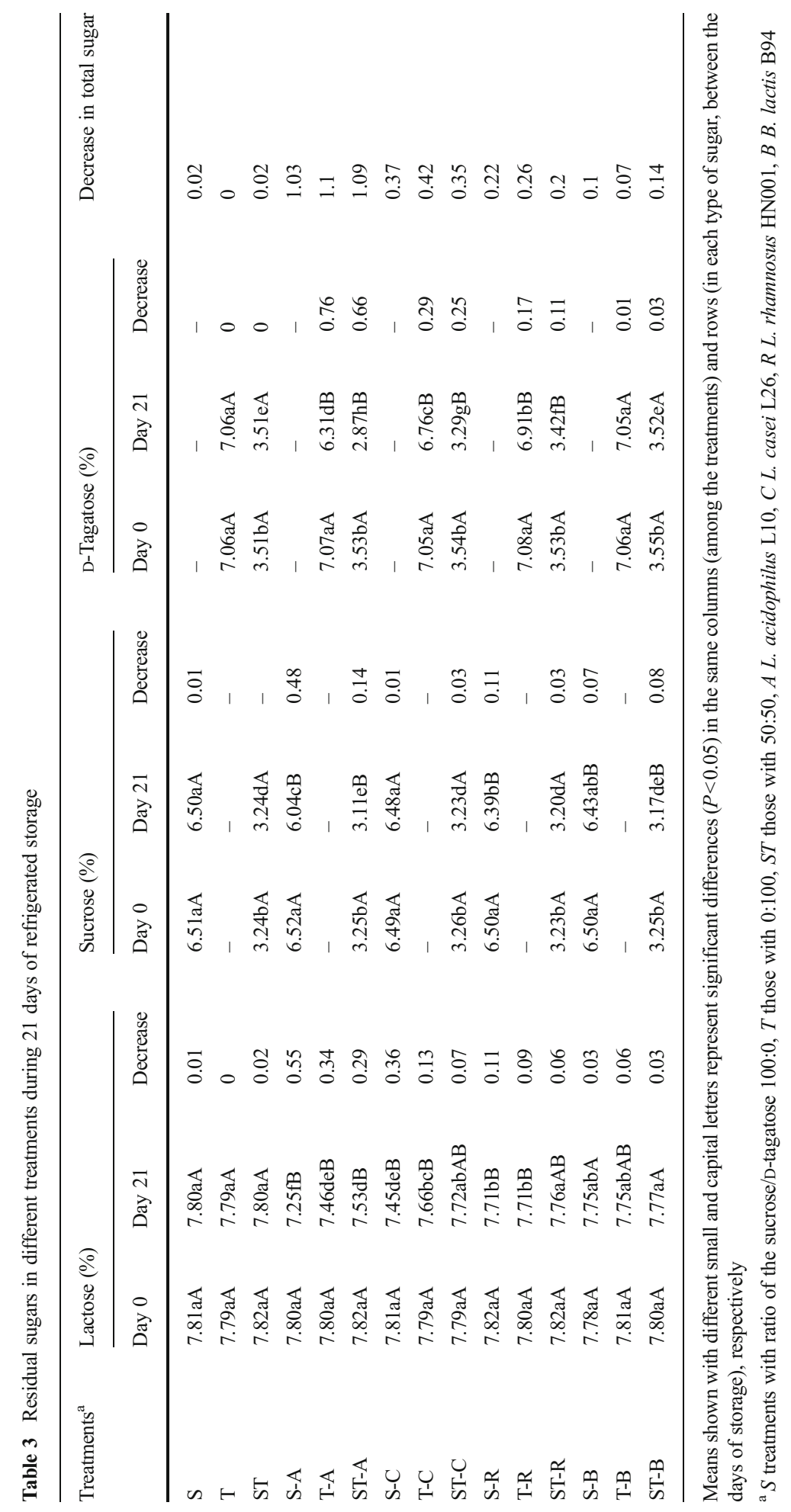


gas chromatographic (GC) analysis of fermented modified MRS broth. They reported that sucrose was used by all strains, except Bifidobacterium bifidum. Also, lactose present in the culture medium ensured growth of each strain of bifidobacteria. However, tagatose was not fermented by any of the used Bifidobacterium strains. Hoyles et al. (2002) reported the results of a polyphasic taxonomic study on some bifidobacteria such as B. lactis CCUG $37979^{\mathrm{T}}$ from human sources. Using API systems, acid was produced from L-arabinose, lactose, mannose, maltose, melibiose, raffinose, ribose, sucrose, and trehalose, but not from D-arabitol, cyclodextrin, pullulan, glycogen, mannitol, methyl $\beta$-D-glucopyranoside, sorbitol, and tagatose. Bertelsen et al. (2001) screened a number of 174 normal or pathogenic human enteric bacteria and dairy lactic acid bacteria for D-tagatose fermentation by incubation for $48 \mathrm{~h}$. Only a few normally occurring enteric human bacteria were able to ferment D-tagatose, among which Enterococcus faecalis, Enterococcus faecium, and Lactobacillus strains can be mentioned. D-tagatose fermentation seems to be common among lactic acid bacteria. According to Bertelsen et al. (2001), most of the analyzed dairy lactic acid bacteria fermented D-tagatose, and among which Lactobacillus, Leuconostoc, and Pediococcus strains most strongly fermented this sugar; after them, Enterococcus, Streptococcus, and Lactococcus were placed. None of the analyzed Bifidobacterium strains such as B. lactis BB-12 fermented tagatose. However, Alander et al. (2001) reported that B. lactis BB-12 weakly fermented tagatose.

Among probiotic chocolate milk, the lowest and greatest consumption of total sugars during 21 days of refrigerated storage were in treatments T-B and T-A, respectively. Also, according to Fig. 1a-c and Table 1, these treatments had the lowest and highest biochemical changes among probiotic treatments at the end of storage, respectively. Therefore, there was a correlation between total consumed sugars and produced organic acids, which was in agreement with the findings of Garro et al. (1999). In probioticcontaining treatments, the highest decrease in lactose, sucrose, and D-tagatose was related to treatments $\mathrm{S}-\mathrm{A}, \mathrm{S}-\mathrm{A}$, and $\mathrm{T}-\mathrm{A}$, and the lowest decrease of the mentioned sugars was for S-B and ST-B, S-C, and T-B, respectively.

\subsection{Color properties during refrigerated storage}

Table 4 shows parameters of color scales in all the treatments during the refrigerated storage. As represented by this table, all the probiotic treatments showed more $L, b$, and $C$ values than non-probiotic ones on day $0(P<0.05)$. Therefore, initial inoculation of probiotics at concentration of $10^{8}$ cfu. $\mathrm{mL}^{-1}$ affected these color scales. It would be noted that whiteness of milk results from scattering visible light by suspended particles, mostly casein micelles (Owens et al. 2001). Also, bacterial cells present at high concentrations (e.g., $10^{7}$ cfu. $\mathrm{mL}^{-1}$ ) might act as light-scattering particles (Owens et al. 2001) and increase $L$ value. The present results were in agreement with those of Owens et al. (2001), who showed that both bacteria-treated milks (with Lactococcus lactis ssp. lactis or Propionibacterium freudenreichii ssp. shermanii) had significantly higher $b$ values than nonfat milk, suggesting a shift from blue to more yellow hues. In the present results, $a$ values in the treatments inoculated with each probiotic bacteria showed a special pattern compared to non-probiotic ones at the start of storage. Owens et al. (2001) suggested that bacterial cell characteristics, such as morphology, could influence color attributes of milk, namely $a$ values. 


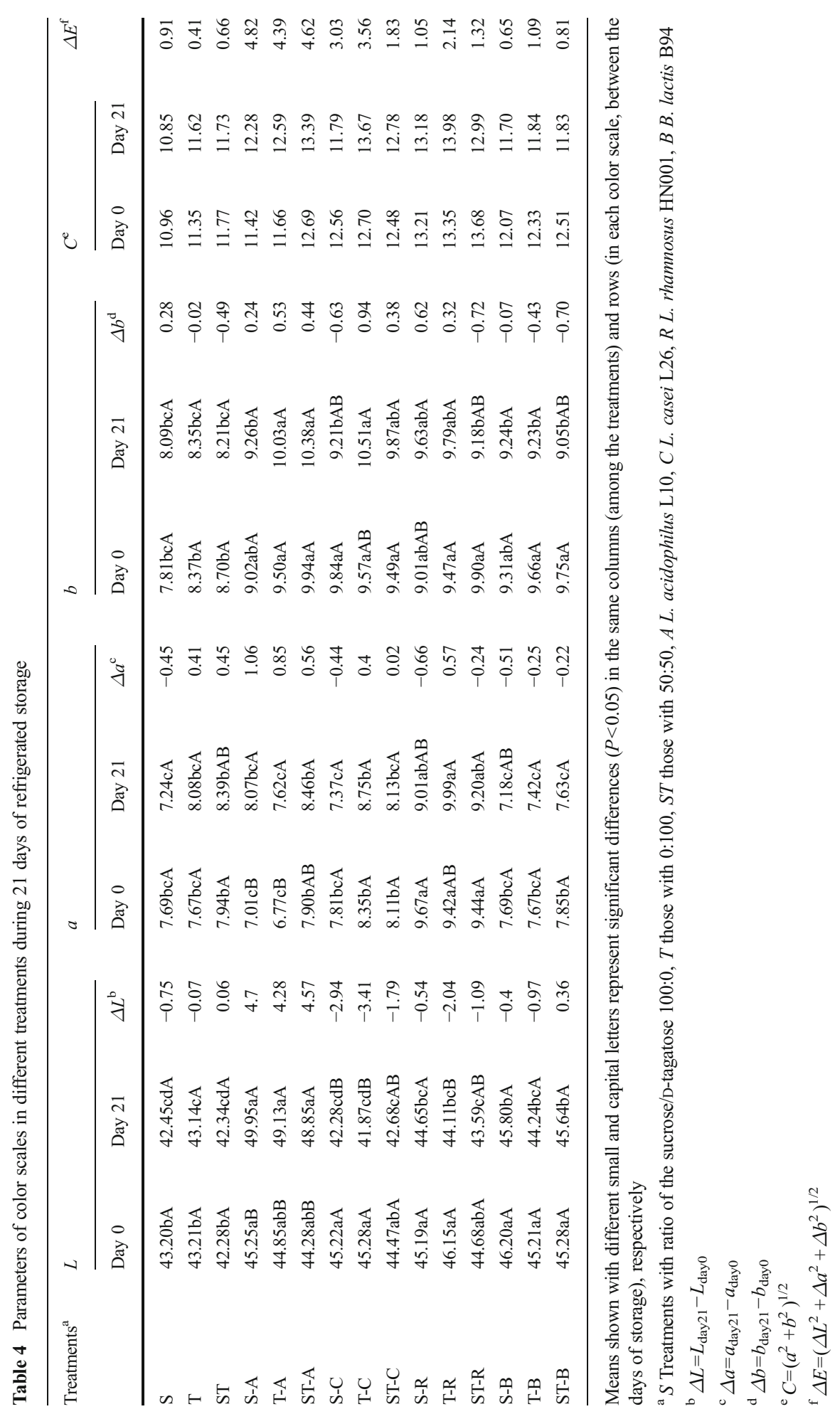

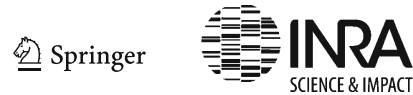


$L, a$, and $b$ values were stable in non-probiotic chocolate milk and those with $B$. lactis during storage $(P>0.05) . L, a$, and $C$ values increased in the treatments inoculated with $L$. acidophilus during the storage and no change occurred in $b$ value. However, those with $L$. casei or L. rhamnosus showed decrease in $L$ value with no changes in $a$ and $b$ values. The cause might be different final $\mathrm{pH}$ in different treatments during the storage. Rankin and Brewer (1998) compared inoculated and fermented nonfat milks $(\mathrm{pH} 4.4)$ to nonfat, $2 \%$ milk fat, and whole milk with instrumental and sensory color measurements. They concluded that $L$ and $a$ values for fermented nonfat milks were higher than nonfat milk, but fermentation had no effects on $b$ value. Also, a trained sensory panel scored whiteness of fermented milks as whiter than nonfat milk. Owens et al. (2001) reported that $L$ values in milks at $\mathrm{pH} 4.0$ and 4.6 were higher than the nonfat milk and $\mathrm{pH} 5.0$ and 6.0 samples. Also, nonfat milks acidified to $\mathrm{pH} 5.0$ displayed the lowest $L$ values. According to the present results, $\mathrm{pH}$ of all the treatments inoculated with L. acidophilus was near 4.6, but those with L. casei or L. rhamnosus were above 5. At $\mathrm{pH}$ of about 4.6, isoelectric point of the casein micelle was reached, resulting in self-association of micelles. Therefore, average particle size sufficiently increased to alter light-reflecting properties (Walstra 1990).

Decrease in $L$ value at $\mathrm{pH}$ of about 5.0 is most likely attributable to decreased size of particles contributed by the altered casein micelles. The mentioned range of $\mathrm{pH}$ represents the one at which colloidal calcium phosphate from the casein micelle structure is maximally solubilized into aqueous phase, allowing for dissociation of casein micelle protein subunits into serum phase of milk and increases porosity of casein micelles (Guinee et al. 1993). Roefs et al. (1985) demonstrated a casein micelle size minima at $\mathrm{pH} 5.2$, resulting from dissociation of several casein protein fractions from the micelle structure due to increased solubility at this $\mathrm{pH}$. Both $a$ and $b$ values in the treatments with $L$. casei or L. rhamnosus were stable and relatively unaffected by dissolution of calcium phosphate from the casein micelle at $\mathrm{pH}$ 5.0. However, $a$ value increased in those with L. acidophilus, which was probably affected by self-association of the casein micelles at $\mathrm{pH}$ near isoelectric point of 4.6. These results were in contrast with the findings by Owens et al. (2001).

Ratio of sucrose/D-tagatose did not show any single effect on color scales during the refrigerated storage and its effects were in combination with effects of type of inoculated probiotics. According to Table 4, the treatments inoculated with L. acidophilus, L. casei, L. rhamnosus, B. lactis, and non-probiotic ones showed the highest to lowest $\Delta E$ value, respectively. It is interesting to note that this order was similar to that of biochemical changes (Section 3.1). Rankin and Brewer (1998) also reported that $\Delta E$ values for the fermented milks were higher than the non-fermented ones.

As shown in Table 4, $L$ and $a$ values showed more changes during the storage than $b$ value. Simultaneous evaluation of $L$ and $a$ values can be accomplished by a lightnessredness diagram. Figure 2 shows relative spatial positions of different chocolate milk on lightness-redness diagram at the start and end of the refrigerated storage. Examination of the present data in that manner (Fig. 2) demonstrated relative spatial positions of chocolate milk samples from color point of view, in which size of arrows indicated changes in $L$ and $a$ values during the storage. Therefore, the treatments inoculated with $L$. acidophilus had the most changes in both $L$ and $a$ values. 

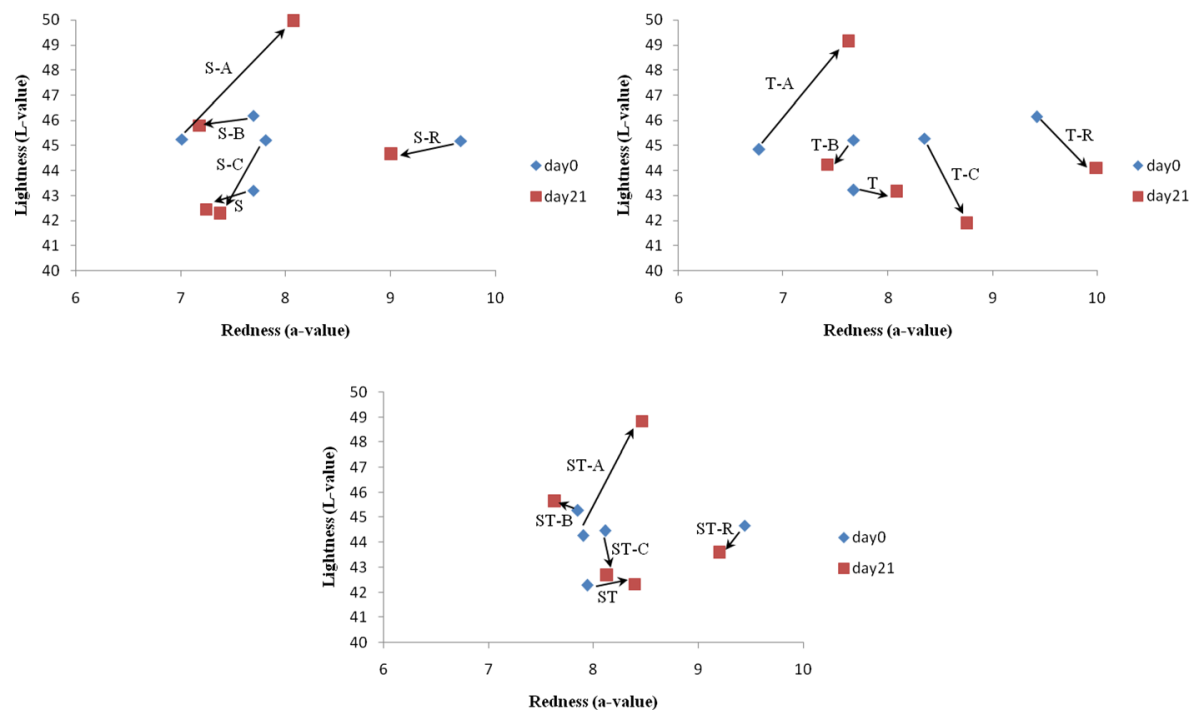

Fig. 2 Relative spatial positions of different chocolate milks on the lightness-redness diagram at the start and end of refrigerated storage

\subsection{Sensory evaluation}

Figure 3 indicates sensory characteristics of different chocolate milk (flavor, mouthfeel, appearance, and total score) at the start and end of the refrigerated storage. The panelists were unable to distinguish differences in color properties among the treatments and accepted all of them from color point of view (data not shown). As shown in Fig. 3, none of the treatments were significantly different in sensory characteristics at the start of storage $(P>0.05)$. Different ratios of sucrose/D-tagatose had no significant effect on sensory characteristics during the storage; however, type of inoculated probiotic had such an effect. The highest sensory scores at the end of storage belonged to control chocolate milk and then to those inoculated with L. rhamnosus and B. lactis. Therefore, these treatments showed acceptable shelf life for 21 days at $5{ }^{\circ} \mathrm{C}$. However, the treatments inoculated with L. acidophilus and L. casei had the lowest scores and even were not considered suitable for drinking at the end of storage. High acidity and low $\mathrm{pH}$ of these probiotic treatments led to acidic taste, visible coagulated particles, and
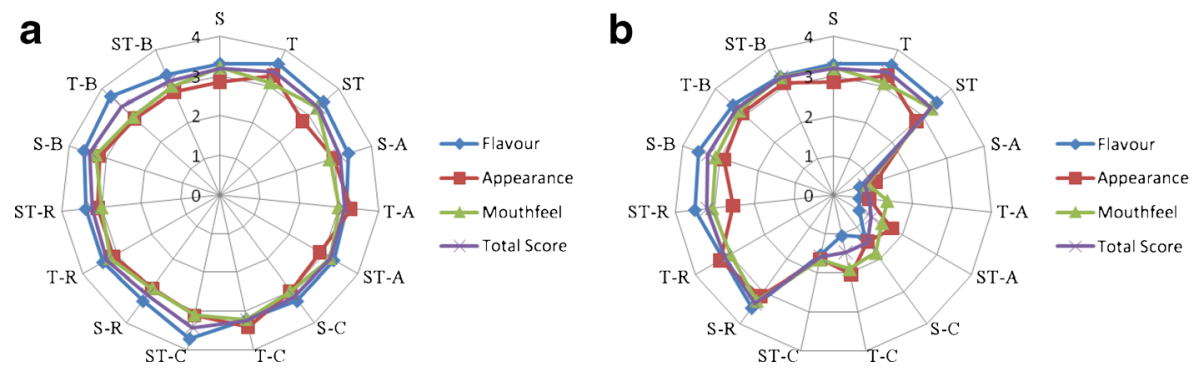

Fig. 3 Sensory characteristics of different chocolate milks at the start (a) and the end (b) of refrigerated storage 
undesirable mouthfeel. Effect of titrable acidity on perception of sourness taste is more important than $\mathrm{pH}$ (Belitz et al. 2009). According to Fig. 3 and Table 1 (final titrable acidity levels), the panelists were able to perceive unpleasant sourness and other unfavorable sensory characteristics related to titrable acidity at value of $33.2^{\circ} \mathrm{D}$ at the end of storage (ST-C and S-C treatments), but not at $27.6^{\circ} \mathrm{D}$ (T-R treatment). Therefore, if the limit of $0-27.6^{\circ} \mathrm{D}$ was considered a recognition limit, treatments T-A and ST-A would be acceptable only for 7 days of refrigerated storage, S-A and all chocolate milk inoculated with $L$. casei for 14 days and S-R, and ST-R and those inoculated with bifidobacteria for 21 days.

\section{Conclusion}

Results of this study revealed that type of probiotic strains and replacement of sucrose with D-tagatose significantly $(P<0.05)$ affected viability of probiotic bacteria as well as physical and chemical characteristics of chocolate milk during 21 days of the refrigerated storage. Sensory attributes of the samples were affected by only type of probiotic strains. The treatments inoculated with L. acidophilus, L. casei, L. rhamnosus, and $B$. lactis as well as non-probiotic ones showed the most to the least biochemical changes and $\Delta E$ value, respectively. The greatest viability throughout the storage time was related to D-tagatose with L. acidophilus (T-A) until day 14 of storage and then to D-tagatose with L. casei (T-C) as well as D-tagatose with L. rhamnosus (T-R) until day 21. Although L. acidophilus, L. casei, and L. rhamnosus mostly tended to ferment Dtagatose, B. lactis did not significantly affect its consumption. The highest sensory scores at the end of storage belonged to control chocolate milk and then to those inoculated with L. rhamnosus and B. lactis. In general, treatments T-R, ST-R, T-B, and ST-B were found to be optimal for industrial production considering total aspects including probiotic viability, functional property of D-tagatose, and sensory attributes. Investigations using other probiotic species and strains as well as other low-calorie and/ or prebiotic sweeteners are recommended as complementary studies.

Acknowledgments We are grateful to the Department of Food Science and Technology, National Nutrition and Food Technology Research Institute (Shahid Beheshti University of Medical Sciences) for support of this study. This paper has been resulted from the student M.Sc. thesis of Shahid Beheshti University of Medical Sciences.

\section{References}

Alander M, Mättö J, Kneifel W, Johansson M, Kögler B, Crittenden R, Mattila-Sandholm T, Saarela M (2001) Effect of galacto-oligosaccharide supplementation on human faecal microflora and on survival and persistence of Bifidobacterium lactis Bb-12 in the gastrointestinal tract. Int Dairy J 11(10):817825

Azcarate-Peril MA, McAuliffe O, Altermann E, Lick S, Russell WM, Klaenhammer TR (2005) Microarray analysis of a two-component regulatory system involved in acid resistance and proteolytic activity in Lactobacillus acidophilus. Appl Environ Microbiol 71(10):5794-5804

Bayoumi HM, Mohamed A, El-Sheikh M, Farrag A, Eissa HA (2011) Effect of ultrafiltration permeate on the quality of chocolate milk. J Am Sci 7(7):616-621 
Belitz H, Grosch W, Schieberle P (2009) Food chemistry, 4th edn. Springer, Berlin Heidelberg

Bertelsen H, Jensen BB, Buemann B (1999) D-tagatose - a novel low-calorie bulk sweetener with prebiotic properties. World Rev Nutr Diet 85:98-109

Bertelsen H, Andersen H, Tvede M (2001) Fermentation of D-tagatose by human intestinal bacteria and dairy lactic acid bacteria. Microb Ecol Health Dis 13(2):87-95

Bogdanov S, Baumann E (1988) Bestimmung von Honigzucker mit HPLC. Mitt Geburtshilfe Lebensm Unters Hyg 79:198-206

Drake MA (2009) Modern sensory practices. In: Clark S, Costello M, Drake M, Bodyfelt F (eds) The sensory evaluation of dairy products. Springer, New York, pp 505-530

FDA (2003) Food labeling: health claims; D-tagatose and dental caries. Final rule. Food and Drug Administration, Department of Health and Human Services (HHS), Federal register 68 (128): 39831-39833. http://www.gpo.gov/fdsys/pkg/FR-2003-07-03/html/03-16949.htm. Accessed 21 Mar 2014

Ferdousi R, Rouhi M, Mohammadi R, Mortazavian AM, Khosravi-Darani K, Homayouni Rad A (2013) Evaluation of probiotic survivability in yogurt exposed to cold chain interruption. Iran $\mathrm{J}$ Pharm Res 12(Suppl):137-142

Garro MS, de Valdez GF, Oliver G, de Giori GS (1999) Starter culture activity in refrigerated fermented soymilk. J Food Prot 62(7):808-810

Gilliland S, Lara R (1988) Influence of storage at freezing and subsequent refrigeration temperatures on $\beta$ galactosidase activity of Lactobacillus acidophilus. Appl Environ Microbiol 54(4):898-902

Gueimonde M, Delgado S, Mayo B, Ruas-Madiedo P, Margolles A, de los Reyes-Gavilán CG (2004) Viability and diversity of probiotic Lactobacillus and Bifidobacterium populations included in commercial fermented milks. Food Res Int 37(9):839-850

Guinee T, Pudja P, Farkye N (1993) Fresh acid-curd cheese varieties. In: Fox P (ed) Cheese: chemistry, physics and microbiology, 2nd edn. Springer, London, pp 363-419

Helland MH, Wicklund T, Narvhus JA (2004) Growth and metabolism of selected strains of probiotic bacteria in milk-and water-based cereal puddings. Int Dairy J 14(11):957-965

Hoyles L, Inganäs E, Falsen E, Drancourt M, Weiss N, McCartney AL, Collins MD (2002) Bifidobacterium scardovii sp. nov., from human sources. Int J Syst Evol Microbiol 52(3):995-999

IDF (1992) General standard of identity for fermented milks. Int Dairy Fed 163:1-4

Ishibashi N, Shimamura S (1993) Bifidobacteria: research and development in Japan. Food Technol 46:126-135

JECFA In: Joint FAO/WO Expert Committee on Food Additives, Sixty-Third Meeting 8-17 June, Geneva, 2004. http://www.who.int/ipcs/publications/jecfa/en/Summary63final.pdf. Accessed 21 Mar 2014

Jensen BB, Buemann B (1998) D-Tagatose. The influence of D-tagatose on bacterial composition and fermentation capacity of faecal samples from human volunteers. Internal report of the Research Centre Foulum, Danish Institute of Agricultural Science, for MD Foods amba

Kim P (2004) Current studies on biological tagatose production using L-arabinose isomerase: a review and future perspective. Appl Microbiol Biotechnol 65(3):243-249

Korbekandi H, Mortazavian A, Iravani S (2011) Technology and stability of probiotic in fermented milks containing probiotics and prebiotic. In: Shah N (ed) Probiotic and prebiotic foods: technology, stability and benefits to the human health. Nova Science Publishing Ltd, USA, pp 131-167

Krutmann J (2009) Pre-and probiotics for human skin. J Dermatol Sci 54(1):1-5

Lankaputhra W, Shah N, Britz M (1996) Survival of bifidobacteria during refrigerated storage in the presence of acid and hydrogen peroxide. Milchwissenschaft-Milk Sci Int 51(2):65-69

Levin GV (2002) Tagatose, the new GRAS sweetener and health product. J Med Food 5(1):23-36

Leyer GJ, Li S, Mubasher ME, Reifer C, Ouwehand AC (2009) Probiotic effects on cold and influenza-like symptom incidence and duration in children. Pediatrics 124(2):172-179

Menrad K (2003) Market and marketing of functional food in Europe. J Food Eng 56(2):181-188

Mohammadi R, Mortazavian AM, Khosrokhavar R, da Cruz AG (2011a) Probiotic ice cream: viability of probiotic bacteria and sensory properties. Ann Microbiol 61(3):411-424

Mohammadi R, Rouhi M, Mortazavian A (2011b) Effects of music waves on fermentation characteristics and viability of starter cultures in probiotic yogurt. Milchwissenschaft-Milk Sci Int 66(2): 193-196

Mortazavian A, Khosrokhavar R, Rastegar H, Mortazaei G (2010) Effects of dry matter standardization order on biochemical and microbiological characteristics of freshly made probiotic Doogh (Iranian fermented milk drink). Ital J Food Sci 22(1):98-104 
Mortazavian A, Mohammadi R, da Cruz A (2011) Technology and stability of probiotic and prebiotic dairy desserts. In: Shah N (ed) Probiotic and prebiotic foods: technology, stability and benefits to the human health. Nova Science Publishers Inc, New York, USA, pp 233-252

Nighswonger BD, Brashears M, Gilliland S (1996) Viability of Lactobacillus acidophilus and Lactobacillus casei in fermented milk products during refrigerated storage. J Dairy Sci 79(2):212-219

Owens S, Brewer J, Rankin S (2001) Influence of bacterial cell population and $\mathrm{pH}$ on the color of nonfat milk. LWT-Food Sci Technol 34(5):329-333

Premi L, Sandine W, Elliker P (1972) Lactose-hydrolyzing enzymes of Lactobacillus species. Appl Microbiol 24(1):51-57

Rankin SA, Brewer JL (1998) Color of nonfat fluid milk as affected by fermentation. J Food Sci 63(1):178180

Reid G (2001) Regulatory and clinical aspects of dairy probiotics [online]. Background paper for the Joint FAO/WHO Expert Consultation on Evaluation of Health and Nutritional Properties of Probiotics in Food Including Powder Milk with Live Lactic Acid Bacteria, Rome, Italy. Food and Agriculture Organization of the United Nations (FAO). ftp://ftp.fao.org/es/esn/food/Reid.pdf. Accessed 20 July 2014

Roefs S, Walstra P, Dalgleish D, Horne D (1985) Preliminary note on the change in casein micelles caused by acidification [skim milk]. Neth Milk Dairy J 39:119-122

Rouhi M, Sohrabvandi S, Mortazavian A (2013) Probiotic fermented sausage: viability of probiotic microorganisms and sensory characteristics. Crit Rev Food Sci Nutr 53(4):331-348

Roy D, Ward P (1990) Evaluation of rapid methods for differentiation of Bifidobacterium species. J Appl Bacteriol 69(5):739-749

Rulis AM (2001) Agency Response Letter GRAS Notice No. GRN 000078. US Food and Drug Administration. http:/www.fda.gov/Food/IngredientsPackagingLabeling/GRAS/NoticeInventory/ ucm154191.htm. Accessed 21 Mar 2014

Sasaki M, Bosman BW, Tan PS (1995) Comparison of proteolytic activities in various lactobacilli. J Dairy Res 62(4):601-610

Shah NP (2007) Functional cultures and health benefits. Int Dairy J 17(11):1262-1277

Shin HS, Lee JH, Pestka J, Ustunol Z (2000) Growth and viability of commercial Bifidobacterium spp in skim milk containing oligosaccharides and inulin. J Food Sci 65(5):884-887

Venema K, Vermunt SHF, Brink EJ (2005) D-Tagatose increases butyrate production by the colonic microbiota in healthy men and women. Microbiol Ecol Health D 17(1):47-57

Ventura M, Margolles A, Turroni F, Zomer A, Clara G, van Sinderen D (2011) Stress responses of Bifidobacteria. In: Tsakalidou E, Papadimitriou K (eds) Stress responses of lactic acid bacteria. Springer, New York, pp 323-347

Walstra P (1990) On the stability of casein micelles. J Dairy Sci 73(8):1965-1979

Yanes M, Durán L, Costell E (2002) Rheological and optical properties of commercial chocolate milk beverages. J Food Eng 51(3):229-234 